\title{
Polymer Ligating Clips in Urologic Laparoscopic Surgery
}

\author{
NICOLAE GRIGORE, VALENTIN PIRVUT, IONELA MIHAI, SEBASTIAN IOAN CERNUSCA MITARIU, MIHAI SAVA*, \\ ADRIAN HASEGAN \\ Lucian Blaga University of Sibiu, Faculty of Medicine, 2A Lucian Blaga Str., 550169, Sibiu, Romania
}

\begin{abstract}
Introducing polymer ligating clips (PLC) or Hem-o-lok clips was a useful alternative to titanium clips in order to perform a safe, efficient and rapid ligation of the vascular structures during laparoscopic and/or conventional surgical procedures. In urologic surgery they are useful during ablative laparoscopic surgery for renal tumor, urothelial tumors, non-functional kidney, bladder tumors and prostate tumors. Polymers' properties depend on the structure of molecules and vary from flexible substances to expandable rigid materials. They have a high resistance to shock, considerable abrasion resistance and precise durability. Polymers can easily take the desired shape, due to different processing modes. They also have the property of resistance to sustainable action of external forces. The aim of this paper is to evaluate the efficacy and safety of PLC used during renal pedicle ligation in retroperitoneal laparoscopic nephrectomy for renal tumors, high urothelial tumors and nonfunctional kidney.
\end{abstract}

Keywords: polymers ligating clips, Hem-o-lok, nephrectomy, laparoscopy, renal tumor, urothelial tumor

The first laparoscopic nephrectomy was reported by Clayman et al. and since then laparoscopic approach has been steadily increasing [1]. Laparoscopic surgery is currently widely used in urologic pathology. Laparoscopic nephrectomy is nowadays a recommended procedure for renal tumors stages T1and T2, with equivalent results to conventional techniques. Nephroureterectomy can also be performed laparoscopically with equivalent oncological results as open surgery [2]. From technical point of view laparoscopic nephrectomy is considered difficult due to surgical risk management of the vascular pedicle. Most surgeons use titanium or polymer ligating clips to control the vascular pedicle.

The launch of PLC under the name of Hem-o-lok in 1999 by Ethicon part of Johnson \& Johnson, was a useful alternative to titanium clips, endoscopic staplers and also endoloops [3]. The mechanical properties of polymers depend on the structure of molecules also vary from rigid substances to flexible and extensible material [4]. They have a high resistance to shock, considerable abrasion resistance and also appreciable durability. Polymers can easily take the desired shape, due to different processing modes. They have the property to suffer sustainable deformation under the action of external forces. They are lightw eight materials and are almost 2 times lighter than aluminum $[5,6]$.

There are four dimensions, from $2 \mathrm{~mm}$ to $16 \mathrm{~mm}$ (size S-small, M-medium, L-large, XL-extralarge) of PLC that can be used for ligation of anatomical structures during laparoscopic surgery. Depending on their size PLC can ligate up to $10 \mathrm{~mm}$ of tissue using a laparoscopic clip applier through a $5 \mathrm{~mm}$ trocar and up to $16 \mathrm{~mm}$ of tissue through a $10 \mathrm{~mm}$ trocar. These locking clips are nonresorbable polymer, inert, non-toxic, non-conductive also compatible with CT or MRI scan.

PLC have a curved design that allows expansion in thick tissue and increase their power by closing thinner tissue. Blocking characteristics and the presence of teeth in jaws ensures a safe closure (fig.1). Charging the applicator clip is easy: it has a flexible mechanism that prevents its loss. The closure of the clip is confirmed by tactile and audible feedback. Each cartridge contains 6 clips. A special adhesive tape to the bottom adhere to any sterile surface for a greater load of applicator. PLC allow

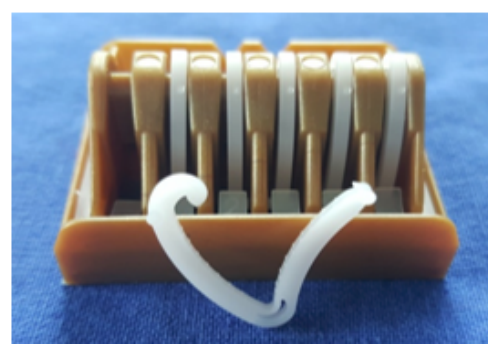

Fig.1 Polymer ligating clip (PLC-Grena LTD Click'aV Plus ${ }^{\mathrm{T}}$ )

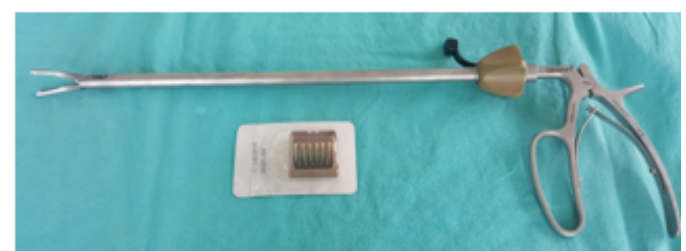

Fig.2 The laparoscopic applicator of PLC

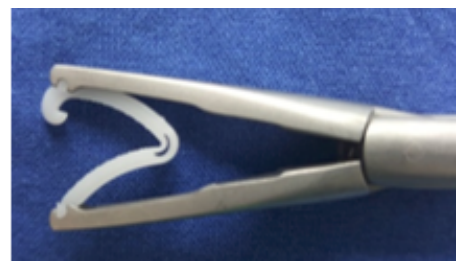

Fig.3 Applicator loaded with clip ready to use

effortlessly loading precision, thus making surgery more effective (fig. 2, 3).

The present paper evaluates the efficacy and safety of PLC used during renal pedicle ligation in retroperitoneal laparoscopic nephrectomy for renal tumors, high urothelial tumors and nonfunctional kidney.

\section{Experimental part}

During 2014-2017 there have been performed in the Urology Department Sibiu 63 laparoscopic nephrectomies through retroperitoneal approach for tumor pathology: 42 radical nephrectomies $(66.6 \%), 13$ nefroureterectomies (20.6\%) for urothelial tumors and 8 simple nephrectomies $(12,7 \%)$ for non-functional kidney. In all cases PLC were used for the renal artery and vein ligation.

Polymer ligating clips used were Grena LTD Click'aV Plus $^{{ }^{M}}$ (fig. 2, 3). They are implantable medical devices made of an advanced polymer to ensure a high level of biocompatibility, with a structural and dimensional stability

\footnotetext{
*email: office@urologiesibiu.ro; Phone: 0745381064
} 


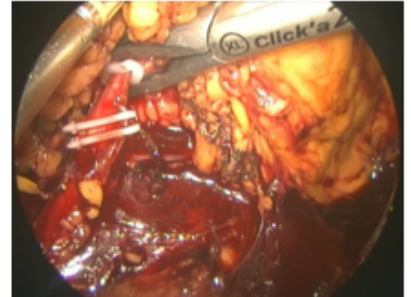

Fig.4 Intraoperative aspects of placing PLC on renal artery

in time, necessary for of long-term safety of the patient [4].

A special attention was paid to the dissection of vascular pedicle. Both the artery and the vein were dissected separately. To reduce the risk of complications, at least 3 $\mathrm{cm}$ of vein must be dissected to allow correct positioning of the clip before ligature of the vein. This can be difficult on the right side, because of the short length of the renal vein.

In all cases there were used three PLC on the artery and three on the vein. There were placed two PLC on the patient side and one on the specimen side. Basically there were used two PLC at the proximal end of the vascular structure for safety reasons: in the case of a sliding clip, the second one provided an efficient hemostasis (fig.4,5).

The PLC failure was defined as the intraoperative or postoperative bleeding that required the replacement of the clip, conversion or re-intervention.

\section{Results and discussions}

The average age of patients was 55.4 (38-82). The study group included 27 women and 36 men.

The Body Mass Index (BMI) ranged from normal weight $(<25)-26 p$, to overweight (25-30) - $20 \mathrm{p}$, obese (30-40) $-7 p$ and morbid obesity $(>40)-10 p$.

Pre-operative tumor stage ranged from T1- $12 p(19.04 \%)$ to T2 - 27 patients (42.85\%) and T3 - $16 \mathrm{p}(25.39 \%)$.

Surgery consisted of radical nephrectomy in $42 p$ $(66.66 \%)$, nephroureterectomy in $13 p(20.36 \%)$ and simple nephrectomy in $8 \mathrm{p}(12.77 \%)$.

Patients were placed in lateral decubitus position under general anethesia. Retroperitoneal access was gained through an incision at the top of twelfth rib with digital dissection to create the workroom. For trocars were used: 3 trocars of $12 \mathrm{~mm}$ and one tocar of $6 \mathrm{~mm}$ The trocars were placed using hand or video control.

No major hemodynamic disorders were found during the surgery. Intraoperative blood loss was approximately $70 \mathrm{~mL}$.

Average time of surgery was $154 \mathrm{~min}$ (90-230 $\mathrm{min})$. The average hospitalization time was 4,1days (from 3 to 7 days). There were used the largest size clips (XL) due to size of vascular elements that have to be ligated (between 5 and $15 \mathrm{~mm}$ ). Patients did not present major vascular complications during the post-operative or post-operative follow-up period (between 3 and 36 months).

Surgical intervention was converted to open surgery in 2 cases (3.17\%) due to significant intraoperative bleeding, 1 patient (1.58\%) needing blood transfusion.

Our results show that the PLC is a safe and effective alternative in the renal pedicle ligation as long as it is properly handled.

Potential causes of the failure of the clip are cited in the literature: animal studies show that the failure of PLC is critically dependent on: the angle of the application of the clip, the ideal angle ranging between 45 and 90 degrees; the ratio between the size of clip and the diameter vessel; blood pressure which can exceed the pressure supported by the clip [7].

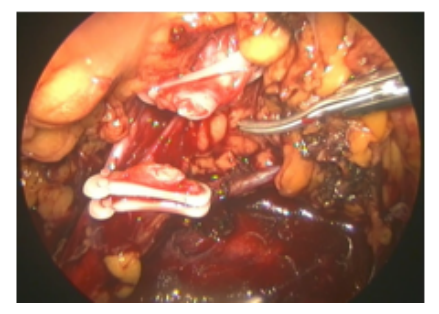

Fig.5 Intraoperative aspects

of cutting the renal artery between the PLC

Surgeons must be familiar with the potential problems that may arise during the handling the renal vascular pedicle and to anticipated the malfunctioning of the devices. Some of the best precautions alreadyknown include: the cautious dissection of vessels, the appropite number, size and position of PLC. Caution in all stages of dissection of the vessels is essential: the vessels need to be nicely dissected from all the fat tissue over a length of at least $3 \mathrm{~cm}$ before applying a PLC. A minimum of two PLC must be applied on the patient side with adequate space between them. The angle between the PLC applicator and the vessel should range from 45 to 90 degrees; any other anplication angles are uncertain. Electrocoagulation near the clips must be avoided in order to prevent tissue necrosis, with slipping or dislocation of the subsequent clip.

Lee Ponsky et al. reported a multiinstitutional review from nine institution with laparoscopic trained urologists who performed 1695 laparoscopic nephrectomies $(899$ radical nephrectomies, 112 simple nephrectomies, 198 nephroureterectomies, 486 donornephrectomies). Follow up was minimum of 6 months from the time of surgery. For each case hem-o-lok clips were used to control the renal artery and the renal vein. The number of clips placed on the patient side of the renal artery was most often 2 , occasionally 3 . The number of clips placed on the patient side of renal vein was most often 2 and rarely 3 . All cases used the large $(L)$ clip on the artery, and most cases of renal vein used the extra-large (XL). No case of clip failure such as intraoperative clip dislodgement necessitating reoperation was recorded [8].

Another report from Sanjay Gandhi Postgraduate Institute of Medical Sciences, Lucknow, India (Kapoor R et al) included 246 laparoscopic ablative nephrectomies performed for benign and malignant condition, which underwenteither standard trasperitoneal or retroperitoneal nephrectomy. Venous and arterial control were employed to secure the occlusion. The features compared were the number of clips used, safety, costand requirementfor blood transfusion. Conversion to an open surgery was required in $36 \mathrm{p}$. In all cases, arterial and venous control was achieved by application of two hem-o-lok clips on the patient side. Blood transfusion was required by $7.2 \%$ of the patients, but none was attributable to clip-related complication [9].

Izaki et al reported 40 laparoscopic nephrectomies in which renal pedicle ligation was accomplished using extralarge (XL) hem-o-lok clips on both the renal arteries and veins by placing two clips on the patient side and one clip on the specimen side. Vascular control using XL hemo-lok clips was successful in al 40 cases without any slipping of clips or uncontrolled bleeding [10].

\section{Conclusions}

Polymer ligating clips are a safe and effective alternative in ligation of vascular structures during laparoscopic surgery in urology.

Using the appropriate size of the PLC according to the vascular structure diameter, provides a safe ligation , without the risk of secondary hemorrhage.

Appropriate vascular dissection, separation of the vascular elements which must be tied and application of 
at least two clips on in the proximal end of the vascular structures are steps to be followed to prevent bleeding with potentially fatal risc both intra and post-operative period.

Acknowledgements: This study, being a retrospective one, did not require a written consent from the patients involved. The authors declare no conflict of interests and no sponsorship was provided by the manufacturer of the clips involved in this study. All authors have read and approved this publication and had equal scientific contribution in publishing this material.

\section{References}

1.CLAYMAN RV, KAVOUSSI LR, SOPER NJ, et al. Laparoscopic nephrectomy: initial case report. J.Urol.1991, 146:278-82.

2.*** EUROPEAN ASSOCIATION OF UROLOGY GUIDELINES 2017 edition: Renal Cell Carcinoma: page 22-37

3.MENG MV. Reported failures of the polymer self-locking (Hem-0lok) clip: review of data from the Food and Drug Administration.J Endourol. 2006;20(12):1054-7.

4.ROBERT J. YOUNG, PETER A. LOWELL, Introduction to Polymers, Third Edition 2011, Chapter 1 page 9-14, Chapter 2 page 15-18. 5 PAUN, V.P., CIMPOESU, N., HANU CIMPOESU, R., MUNCELEANU, G.V., FORNA, N., AGOP, M., On the Energy Dissipation Capacity and the Shape Memory. A Comparative Study between Polymer Composites and Alloys, Mat.Plast., 47, nr. 2 , 2010, p158
6 LAZAR,M., FIAT , D., BACIU, V., HUBCA, Gh., Researches Regarding the Influence of Some Concrete Protection Polymeric Products, Mat.Plast., 49, no. 1, 2012,p 9

7.PRASANNA SOORIAKUMARAN, SASHI S. KOMMU, JOANNE COOKE, STHEPHEN GORDON, CHRISTIAN BROWN, BEN EDDY, PETER D. RIMINGTON, ABHAYRANE. Evaluation of a commercial vascular clip: risk factors and predictors of failure from in vitro studies 2008, BJ U International, Volume 103, Version of Record online: 19 Nov.2008. 8.PONSKY LEE, CHERULLO EDWARD, MOINZADEHALIREZA, DESAI MIHIR, KAOUK JIHAD, HABER GEORGES-PASCAL, et al. The Hem-olok clip is safe for laparoscopic nephrectomy:A multi-institutional review. Urology Apr. 2008, 71 (4): 593-96.

9.KAPOOR R, SINGH KJ, SURI A, DUBEY D, MANDHANI A, SRIVASTAVA $A$, et al. Hem-o-lok clips for vascular control during laparoscopic ablative nephrectomy: A single-center experience. J endourol Mar 2006, 20(3):202-04.

10.IZAKI H, FUKUMORI T, TAKAHASHI M, NAKATSUJI H, OKA N, TAUE $\mathrm{R}$, et al. Clinical research of renal vein control using hem-0-lok clips in laparoscopic nephrectomy. Int J Urol Aug 2006,13(8):1147-49.

Manuscript received: 24.11 .2016 